\title{
Several New Types of Fixed Point Theorems and Their Applications to Two-Point Ordinary Differential Equations
}

\author{
Congjun Zhang ${ }^{1}$, Jinlu Li $^{2}$, Yan Zhang ${ }^{1}$, Xiaoliang Feng ${ }^{3}$ \\ ${ }^{1}$ School of Applied Mathematics, Nanjing University of Finance and Economics, \\ Nanjing, China \\ ${ }^{2}$ Department of Mathematics, Shawnee State University, Portsmouth, USA \\ ${ }^{3}$ Department of Mathematics, Nanjing University, Nanjing, China \\ Email: zcjyysxx@163.com, jli@shawnee.edu, recluseng@yahoo.com
}

Received November 1, 2010; revised September 3, 2012; accepted September 10, 2012

\begin{abstract}
The present paper is mainly concerned with several new types of fixed point theorems in different spaces such as cone metric spaces and fuzzy metric spaces. By using these obtained fixed point theorems, we then prove the existence and uniqueness of the solutions to two classes of two-point ordinary differential equation problems.
\end{abstract}

Keywords: Expansive Mapping; Cone Metric Space; Fuzzy Metric Space; Two-Point Ordinary Differential Equations

\section{Introduction}

The theory of the fixed point has important applications in fields such as differential equations, equilibrium problems, variational inequality, optimization problems, maxmin problems etc. (cf. Klaus Deimling [1], Congjun Zhang [2] for example), which has attracted many scholars' attention and became a hot topic in mathematics and applied mathematics field for a long time. In recent decades, many new types of fixed point theorems have been proposed (see [3-6] and the reference therein) and the generalizations of the existing ones have been dramatically developed in many ways. In [7], LongGuang Huang and Xian Zhang have introduced the notion of cone metric spaces and proved some fixed point theorems of contractive mappings on cone metric spaces. For fixed point theorems in fuzzy metric spaces, see [8-12]. In [13-16], some scholars have proved the fixed point theorem in partial order metric space, and applied them to prove the existence and uniqueness of the solution to the two-point ordinary differential equation problems. Inspired by the recent progress in this fields, we will study in the present paper the existence and uniqueness of the fixed point for some special mappings in cone metric spaces and fuzzy metric spaces as well as their applications to the following two-point ordinary differential equations.

Problem (1):

$$
\left\{\begin{array}{l}
u^{\prime}(t)=f(t, u(t)), \quad t \in[0, T] . \\
u(0)=u(T),
\end{array}\right.
$$

where $T>0, f: I \times R \rightarrow R$ is a continuous function satisfying some conditions which will be given explicitly later.

\section{Problem (2):}

$$
\left\{\begin{array}{l}
x^{\prime}(t)=f(t, x)+g(t, x), \quad t \in[0, T] \\
x(0)=x_{0},
\end{array}\right.
$$

where $T>0, f: I \times R \rightarrow R$ is a continuous function satisfying some conditions which will be given explicitly later.

The paper is organized as follows. For the reader's convenience, we recall in Section 2 some definitions and lemmas in cone metric spaces and fuzzy metric spaces that will be used in the sequel. Section 3 is devoted to the investigation on the existence and uniqueness of the fixed point for some special mappings in cone metric spaces and fuzzy metric spaces. In last section, two-point ordinary differential equation problems are studied by using the results obtained in Section 3 and the existence and uniqueness of the solutions to such equations is established.

\section{Preliminaries and Abstract Results}

We recall in this section some definitions and lemmas in cone metric spaces and fuzzy metric spaces that will be used in the sequel.

Definition 1 [6]. Let $(X, d)$ be a metric space and $f$ a mapping from $X$ to $X$. For any $z \in X$, define $f^{0} z=z, \quad f^{n} z=f\left(f^{n-1} z\right)$ for $n \geq 1$. The sequence $\left\{f^{n}\right\}$ is called the orbit of $f$ and $f^{n}$ the $n$ iterate of $f$. 
Definition 2. A function $f:[0, \infty) \rightarrow[0, \infty)$ is called an $\omega$-function if it is a monotone increasing function and satisfies that $f(0)=0$ and for any $\varepsilon>0$, there exists $M>0$, such that $f(t)<\varepsilon$, for every $t \in(\varepsilon, M)$.

For example: $f(x)=(1 / 10) x$, defined on $[0, \infty)$, is an $\omega$-function.

Definition 3 [7]. Let $X$ be a nonempty set. Let $E$ be a real Banach space, $K$ a cone of $E$ satisfying $\operatorname{int} K \neq \varnothing$, where $\operatorname{int} K$ denotes the interior of $K$. Define a partial order $\succeq$ on $E$ based on $K$ as follows: for any $x, y \in E, \quad y \succeq x$ if and only if $y-x \in K$, while $y \succ x$ means $y \succeq x$ and $y \neq x$, and $y \succ \succ x$ means $y-x \in \operatorname{int} K$. And the following convention is assumed: $x \preceq y$ if and only if $y \succeq x, x=y$ if and only if $x \preceq y$ and $y \preceq x$.

If a mapping $d: X \times X \rightarrow E$ satisfies:

1) $d(x, y) \succeq 0$, for all $x, y \in X, d(x, y)=0$ if and only if $x=y$;

2) $d(x, y)=d(y, x)$, for all $x, y \in X$;

3) $d(x, y) \preceq d(x, z)+d(z, y)$, for all $x, y, z \in X$, then $d$ is called a cone metric on $X$ and $(X, d)$ is called a cone metric space with respect to the Banach space $E$ and the cone $K$ in $E$.

Definition 4 [2]. 1) A cone $K$ in a Banach space $E$ is called normal, if there exists a number $M>0$ such that for all $x, y \in K, \quad \theta \preceq x \preceq y$ implies $\|x\| \leq M\|y\|$, where $\theta$ is the zero element of the Banach space $E$. The smallest $M$ satisfying that inequality is denoted by $M^{*}$, and it is called the normal constant of $K ; 2$ ) A cone $K$ in a Banach space $E$ is called regular if every increasing sequence which is bounded from above is convergent. That is, if $\left\{x_{n}\right\}$ is sequence such that $x_{1} \leq x_{2} \leq \cdots \leq x_{n} \leq \cdots \leq y$ for some $y \in E$, then there is $x \in E$ such that $\left\|x_{n}-x\right\| \rightarrow 0(n \rightarrow \infty)$.

Remark 1. 1) For any normal cone $K$ in a Banach space $E, M^{*}$ exists and $M^{*} \geq 1$ (see [2]); 2) Equivalently, a cone $K$ is regular if and only if every decreasing sequence which is bounded from below is convergent. It is well known that a regular cone is a normal cone.

Definition 5 [7]. Let $(X, d)$ be a cone metric space with respect to a Banach space $E$ and a cone $K$ in $E$. Let $\left\{x_{n}\right\}$ be a sequence in $X$ (see [7]).

1) $\left\{x_{n}\right\}$ is called a convergent sequence with limit $x$, if for any $c \in E$, there exists $n_{0} \in N$ such that for every $n>n_{0}, d\left(x_{n}, x\right) \prec \prec c$ holds. In this case, we denote the limit of $\left\{x_{n}\right\}$ by $\lim _{n \rightarrow \infty} x_{n}=x$, or

$x_{n} \rightarrow x(n \rightarrow \infty)$.

2) $\left\{x_{n}\right\}$ is called a Cauchy sequence on $X$, if for any $c \in E$ with $0 \prec c$, there exists $n_{0} \in N$ such that for each $m, n>n_{0}, d\left(x_{n}, x_{m}\right) \prec \prec c$ holds.

3) We call $X$ a complete cone metric space with respect to the Banach space $E$ and the cone $K$ in $E$, if every Cauchy sequence is convergent in $X$.
Remark 2. If $K$ is a normal cone, then $\left\{x_{n}\right\}$ converges to $x$ if and only if $d\left(x_{n}, x\right) \rightarrow \theta$, as $n \rightarrow \infty$. $\left\{x_{n}\right\}$ is a Cauchy sequence on $X$ if and only if $d\left(x_{n}, x_{m}\right) \rightarrow \theta$ as $m, n \rightarrow \infty$ (see[7]).

Definition 6 [7]. Let $(X, d)$ be a cone metric space with respect to a Banach space $E$ and a cone $K$ in $E$. If for any sequence $\left\{x_{n}\right\}$ in $X$, there exists a subsequence $\left\{x_{n_{k}}\right\}$ of $\left\{x_{n}\right\}$, such that $\left\{x_{n_{k}}\right\}$ is convergent in $X$. Then the cone metric space $(X, d)$ is said to be sequentially compact.

Definition $7[9,10]$. A binary operation $*:[0,1]^{2} \rightarrow[0,1]$ is called a continuous $t$-norm, if the following conditions are satisfied: 1$) *$ is associative and commutative; 2$) *$ is continuous; 3) $a * 1=a$ for all $a \in[0,1]$; 4)

$a * b \leq c * d$ whenever $a \leq c$ and $b \leq d$, for each $a, b, c, d \in[0,1]$. If it only satisfies conditions 1$), 2)$ and 4 ), then it is called a $t$-norm.

Four typical examples of continuous $t$-norms are

$a *_{1} b=\min \{a, b\}, a *_{2} b=\frac{a b}{\max \{a, b, \lambda\}}$ for

$0<\lambda<1$ and $a *_{3} b=a b, a *_{4} b=\max \{a+b-1,0\}$.

Definition $8[9,10]$. Let $X$ be an arbitrary nonempty set. Let $*$ be a continuous $t$-norm and $M$ a fuzzy set on $X^{2} \times(0, \infty)$. If the following conditions satisfy:

1) $M(x, y, t)>0$;

2) $M(x, y, t)=1$ if and only if $x=y$;

3) $M(x, y, t)=M(y, x, t)$;

4) $M(x, y, t) * M(y, z, s) \leq M(x, z, t+s)$;

5) $M(x, y,):.(0, \infty) \rightarrow[0,1]$ is continuous, for any $x, y, z \in X$ and $t, s>0$, then the 3-tuple $(X, M, *)$ is called a fuzzy metric space.

Remark 3. For any $x, y \in X$, $M(x, y,):.(0, \infty) \rightarrow[0,1]$ is a non-decreasing function (see $[9,10])$.

Definition $9[9,10]$. Let $(X, M, *)$ be a fuzzy metric space and $M$ a fuzzy set on $X^{2} \times[0, \infty) . M$ is said to satisfies the $n$-property on $X^{2} \times(0, \infty)$ if

$\lim _{n \rightarrow \infty}\left[M\left(x, y, k^{n} t\right)\right]^{n^{p}}=1$ whenever $x, y \in X, k>1$ and $p>0$.

Definition 10. Let $(X, M, *)$ be a fuzzy metric space and $M$ a fuzzy set on $X^{2} \times[0, \infty) . M$ is said to satisfies the $\tau$-property on $X^{2} \times(0, \infty)$ if $\lim _{n \rightarrow \infty} M\left(x, y, k^{n} t\right)=1$ for all $x, y \in X$ and $k>1$.

Definition 11 [11]. A function $f: R^{+} \rightarrow R^{+}$is said to satisfy $\phi$-condition, if $f$ is a strictly increasing function satisfying $f(0)=0$ and $\lim _{n \rightarrow \infty} f^{n}(t)=\infty$ for any $t>0$, where $f^{n}(t)=f\left(f^{n-1}(t)\right)$.

Remark 4. If a function $f: R^{+} \rightarrow R^{+}$satisfies the $\phi$-condition, then the following inequalities hold (see 
[11]):

1) $f(t)>t$, for all $t>0$;

2) $f^{n}(t)>f^{n-1}(t)>\cdots>f(t)>t$, for each $n=1,2, \cdots$ and for all $t>0$.

Definition 12. Let $(X, M, *)$ be a fuzzy metric space, the fuzzy set $M$ is said to have $\phi$-property whenever $\lim _{n \rightarrow \infty} M\left(x, y, f^{n} t\right)=1$ for all $x, y \in X$ where

$f: R^{+} \rightarrow R^{+}$satisfying the $\phi$-condition.

Definition $13[9,10]$. Let $(X, M, *)$ be a fuzzy metric space and $M$ a fuzzy set on $X^{2} \times[0, \infty)$.

1) A sequence $\left\{x_{n}\right\}$ in $X$ is said to fuzzy-convergent to a point $x \in X$, if $\lim M\left(x_{n}, x, t\right)=1$ for all $t>0$.

2) A sequence $\left\{x_{n}\right\}$ in $X$ is called a fuzzy-Cauchy sequence, if for each $0<\varepsilon<1$ and $t>0$, there exists $n_{0} \in N$, such that $M\left(x_{n}, x_{m}, t\right)>1-\varepsilon$ for each $m, n \geq n_{0}$.

3) A fuzzy metric space is called fuzzy-complete, if every fuzzy-Cauchy sequence is fuzzy-convergent.

Definition $14[9,10]$. Let $\left(X, M,{ }^{*}\right)$ be a fuzzy metric space. The fuzzy set $M$ is said to be fuzzy-continuous on $X^{2} \times(0, \infty)$, whenever any $\left\{\left(x_{n}, y_{n}, t_{n}\right)\right\}$ in

$X^{2} \times(0, \infty)$ which fuzzy-converges to

$\{(x, y, t)\} \in X^{2} \times(0, \infty)$ implies

$\lim _{n \rightarrow \infty} M\left(x_{n}, y_{n}, t_{n}\right)=M(x, y, t)$.

Remark 5. $M$ is a continuous function on $X^{2} \times[0, \infty)$ (see $[9,10])$.

Definition 15 [12]. Let $(X, M, *)$ be a fuzzy metric space and $M$ the fuzzy set on $X^{2} \times[0, \infty)$. Denote by $H_{0}(X)$ the set of all compact subsets of $X$ and define a function $H_{M}: H_{0}(X) \times H_{0}(X) \times(0, \infty) \rightarrow(0, \infty)$ by

$$
H_{M}(A, B, t)=\min \left\{\inf _{a \in A} M(a, B, t), \inf _{b \in B} M(A, b, t)\right\}
$$

for any $A, B \in H_{0}(X)$ and any $t>0$, where

$M(a, B, t)=\sup _{b \in B} M(a, b, t)$ and

$M(A, b, t)=\sup _{a \in A} M(a, b, t)$.

Lemma 1 [6]. Let $(X, d)$ be a complete metric space, $f: X \rightarrow X$, for the $n$ iterate of $f \quad(n>1)$, the following statements hold:

1) If $f^{n}$ has a unique fixed point, then $f$ has a unique fixed point.

2) If there exists $z \in X$, such that the orbit of $f^{n}$ converges to $z$, then the orbit of $f$ converges to $z$.

3 ) If the orbit of $f^{n}$ is a bounded sequence, then the orbit of $f$ is a bounded sequence.

Lemma 2. Let $(X, d)$ be a complete metric space and $T$ an expansive and surjective mapping on $X$, then $T$ has a unique fixed point.

Proof. We claim first that $T$ is injective. To show this claim, assume, by the way of contradiction, that there exist $x_{0} \neq y_{0} \in X$ such that $T\left(x_{0}\right)=T\left(y_{0}\right) \in X$. Since $x_{0} \neq y_{0} \in X$, then $d\left(x_{0}, y_{0}\right)>0$ holds. Since $T$ is an expansive mapping, it implies

$d\left(T\left(x_{0}\right), T\left(y_{0}\right)\right)>0$. It contradicts to $T\left(x_{0}\right)=T\left(y_{0}\right)$, that is, $d\left(T\left(x_{0}\right), T\left(y_{0}\right)\right)=0$, which implies $T$ is a bijection. Hence $T^{-1}$ exists and is a contraction mapping. By the contractive mapping priciple, there exists a unique $x_{0} \in X$, such that $T^{-1}\left(x_{0}\right)=x_{0} \in X$, that is $x_{0}=T\left(x_{0}\right) \in X$. The proof is complete.

Lemma 3 [5]. Let $(X, d)$ be a complete metric space and $f$ a self mapping on $X$. If the following condition satisfies, for any $\varepsilon>0$, there exists $\delta>0$, such that $\varepsilon<d(x, y)<\varepsilon+\delta$ implies $d(f(x), f(y))<\varepsilon$, then $f$ has a unique fixed point $\xi$ on $X$, and

$\lim _{n \rightarrow \infty} f^{n}(x)=\xi$ for any $x \in X$.

Lemma 4 [7]. Let $(X, d)$ be a sequentially compact cone metric space with respect to a Banach space $E$ and a regular cone $K$ in $E$. Suppose a mapping $T: X \rightarrow X$ satisfies the contractive condition: $d(T x, T y) \prec d(x, y)$, for all $x, y \in X, x \neq y$, then $T$ has a unique fixed point in $X$.

Lemma 5 [4]. Let $(X, d)$ be a compact metric space and $T$ a self mapping on $X$. Assume that $12 d(x, T x)<d(x, y)$ implies $d(T x, T y)<d(x, y)$ for any $x, y \in X$, then $T$ has a unique fixed point.

Lemma $6[9,10]$. Let $(X, M, *)$ be a fuzzy metric space, $a * b \geq a b$ for all $a, b \in[0,1]$ and $M$ satisfy $n$-property. Let $\left\{x_{n}\right\}$ be a sequence in $X$ such that for all $n \in N, M\left(x_{n}, x_{n+1}, k t\right) \geq M\left(x_{n-1}, x_{n}, t\right)$ for every $0<k<1$, then $\left\{x_{n}\right\}$ is a Cauchy sequence in $X$.

\section{The Existence Theorem of Fixed Points}

In this section, we apply the concepts and lemmas provided in Section 2 to prove some existence theorems of fixed points for some mappings. These results will be used in the following section.

Theorem 1. Let $(X, d)$ be a complete metric space and $f: X \rightarrow X$ a surjective mapping. If there exist $p \in N$ and $h>1$ such that

$$
d\left(f^{p}(x), f^{p}(y)\right) \geq h d(x, y)
$$

holds for any $x, y \in X$, then there exists a unique fixed point of $f$.

Proof. For each $y \in f^{2}(X)$, since $f$ is a surjective, then there exists $z \in f(x)$, such that $f(z)=y$, in the same way, there exist $x \in X$, such that $f(x)=z$, i.e. there exists $x \in X$, such that $f^{2}(x)=y$. We deduce by induction that $f^{p}(x)$ is also surjective, which combining (I) shows that $f^{p}(x)$ is an expansive mapping. By Lemma 2, there exists a unique fixed point of $f^{p}(x)$, then we know by Lemma 1 that there exists a unique fixed point of $f$. The proof is complete.

Remark 6. It is obvious that we can get Lemma 2 
from Theorem 1. An example satisfying Theorem 1 is given below.

Example 1. Define $f: R \rightarrow R$ by

$$
f(x)=\left\{\begin{array}{c}
-\frac{x}{5}, \text { if } x \leq 0 ; \\
-10 x, \text { if } x>0,
\end{array}\right.
$$

it is clear that $f$ is a surjective self-mapping on $R$ and $f^{2}(x)=2 x . f^{2}$ satisfies condition (I), i.e. $p=2$, then $f$ has a fixed point, 0 is the fixed point in this example.

Theorem 2. Let $(X, d)$ be a sequentially compact cone metric space with respect to a Banach space $E$ and a normal cone $K$ in $E$ with normal constant $M^{*}$. Assume that $T$ is a self mapping on $X$ and satisfies for any $x, y \in X, \frac{1}{2 M^{*}}\|\mathrm{~d}(x, T x)\|<\|\mathrm{d}(x, y)\|$ implies $\|\mathrm{d}(T x, T y)\|<\|\mathrm{d}(x, y)\|$, then $T$ has a unique fixed point.

Proof. We claim first that $\beta=0$ where $\beta$ is defined by

$$
\beta=\inf _{x \in X}\|\mathrm{~d}(x, T x)\| .
$$

Using reduction to absurdity, we suppose $\beta>0$. Since $(X, d)$ is sequentially compact, we deduce from the definition of $\beta$ that there exists a sequence $\left\{x_{n}\right\}$ such that

$$
\left\|\mathrm{d}\left(x_{n}, T x_{n}\right)\right\| \rightarrow \beta(n \rightarrow \infty)
$$

and

$$
\left(x_{n}, T x_{n}\right) \rightarrow(\xi, \eta)(n \rightarrow \infty)
$$

for some $\xi, \eta \in X$. Observe that the normal constant $M^{*} \geq 1$, there exists $n_{0}>0$ such that for any $n \geq n_{0}$ the inequality

$$
\frac{1}{2 M^{*}}\left\|\mathrm{~d}\left(x_{n}, T x_{n}\right)\right\|<\left\|\mathrm{d}\left(x_{n}, \eta\right)\right\|
$$

holds, which combining the given conditions shows that for any $n \geq n_{0}$,

$$
\left\|\mathrm{d}\left(T x_{n}, T \eta\right)\right\|<\left\|\mathrm{d}\left(x_{n}, \eta\right)\right\| .
$$

By calculations we then have

$$
\begin{aligned}
\left\|\mathrm{d}\left(T \eta, T^{2} \eta\right)\right\| & <\|\mathrm{d}(\eta, T \eta)\|=\lim _{n \rightarrow \infty}\left\|\mathrm{d}\left(T x_{n}, T \eta\right)\right\| \\
& \leq \lim _{n \rightarrow \infty}\left\|\mathrm{d}\left(x_{n}, \eta\right)\right\|=\beta
\end{aligned}
$$

which contradicts to the definition of $\beta$.

We prove next that $T$ has a fixed point. We proceed once more by using reduction to absurdity and suppose that $T$ has no fixed point. Then for each $n \in N$,

$$
0<\frac{1}{2 M^{*}}\left\|\mathrm{~d}\left(x_{n}, T x_{n}\right)\right\|<\left\|\mathrm{d}\left(x_{n}, T x_{n}\right)\right\|,
$$

which implies that for each $n \in N$,

$$
\left\|\mathrm{d}\left(T x_{n}, T^{2} x_{n}\right)\right\|<\left\|\mathrm{d}\left(x_{n}, T x_{n}\right)\right\| .
$$

By the triangle inequality in cone metric spaces, we have

$$
\mathrm{d}\left(\xi, T^{2} x_{n}\right) \preceq \mathrm{d}\left(\xi, T x_{n}\right)+\mathrm{d}\left(T x_{n}, T^{2} x_{n}\right),
$$

then,

$$
\begin{aligned}
\lim _{n \rightarrow \infty}\left\|\mathrm{d}\left(\xi, T^{2} x_{n}\right)\right\| & \leq \lim _{n \rightarrow \infty} M^{*}\left\|\left(\mathrm{~d}\left(\xi, T x_{n}\right)+\mathrm{d}\left(T x_{n}, T^{2} x_{n}\right)\right)\right\| \\
& \leq \lim _{n \rightarrow \infty} M^{*}\left(\left\|\left(\mathrm{~d}\left(\xi, T x_{n}\right)\|+\| \mathrm{d}\left(x_{n}, T x_{n}\right)\right)\right\|\right) \\
& =M^{*}\|\mathrm{~d}(\xi, \eta)+\mathrm{d}(\xi, \eta)\|=0 .
\end{aligned}
$$

We claim that at least one of the following two inequalities should be hold:

$$
\begin{gathered}
\frac{1}{2 M^{*}}\left\|\mathrm{~d}\left(T x_{n}, T^{2} x_{n}\right)\right\|<\left\|\mathrm{d}\left(T x_{n}, \xi\right)\right\|, \\
\frac{1}{2 M^{*}}\left\|\mathrm{~d}\left(x_{n}, T x_{n}\right)\right\|<\left\|\mathrm{d}\left(x_{n}, \xi\right)\right\|,
\end{gathered}
$$

otherwise, we reach a contradiction by the following calculations:

$$
\begin{aligned}
& \left\|\mathrm{d}\left(x_{n}, T x_{n}\right)\right\| \leq M^{*}\left\|\left(\mathrm{~d}\left(x_{n}, \xi\right)\|+\| \mathrm{d}\left(T x_{n}, \xi\right)\right)\right\| \\
& \leq M^{*}\left(\frac{1}{2 M^{*}}\left\|\mathrm{~d}\left(T x_{n}, T^{2} x_{n}\right)\right\|+\frac{1}{2 M^{*}}\left\|\mathrm{~d}\left(x_{n}, T x_{n}\right)\right\|\right) \\
& <\frac{1}{2}\left\|\mathrm{~d}\left(x_{n}, T x_{n}\right)\right\|+\frac{1}{2}\left\|\mathrm{~d}\left(x_{n}, T x_{n}\right)\right\|=\left\|\mathrm{d}\left(x_{n}, T x_{n}\right)\right\| .
\end{aligned}
$$

If the first inequality of the above two holds, then

$$
\begin{aligned}
\|\mathrm{d}(\xi, T \xi)\| & \leq M^{*}\left(\left\|\mathrm{~d}\left(\xi, T^{2} x_{n}\right)\right\|+\left\|\mathrm{d}\left(T \xi, T^{2} x_{n}\right)\right\|\right) \\
& \leq M^{*}\left(\left\|\mathrm{~d}\left(\xi, T^{2} x_{n}\right)\right\|+\left\|\mathrm{d}\left(\xi, T x_{n}\right)\right\|\right) \\
& \rightarrow 0(n \rightarrow \infty),
\end{aligned}
$$

if the the other one holds, then

$$
\begin{aligned}
\|\mathrm{d}(\xi, T \xi)\| & \leq M^{*}\left(\left\|\mathrm{~d}\left(\xi, T x_{n}\right)\right\|+\left\|\mathrm{d}\left(T \xi, T x_{n}\right)\right\|\right) \\
& \left.\leq M^{*}\left(\left\|\mathrm{~d}\left(\xi, T x_{n}\right)\right\|+\left\|\mathrm{d}\left(\xi, x_{n}\right)\right\|\right)\right] \\
& \rightarrow 0(n \rightarrow \infty),
\end{aligned}
$$

which show that $T \xi=\xi$ in each case, and the proof of the existence of the fixed point is complete.

We finally prove the uniqueness of the fixed point. Suppose $v \in X, v \neq u$ and $T v=v$. Since

$\frac{1}{2 N^{*}}\|\mathrm{~d}(u, T u)\|=0<\|\mathrm{d}(u, v)\|$, then

$\|\mathrm{d}(u, T v)\|=\|\mathrm{d}(T u, T v)\|<\|\mathrm{d}(u, v)\|$, we reach a contradiction which completes the proof.

Remark 7. In [7], Long-Guang Huang and Xian Zhang have established a fixed point theorem in a se- 
quentially compact cone metric space with respect to a Banach space $E$ and a regular cone $K$ in $E$ (see Lemma 4), where the mapping $T: X \rightarrow X$ satisfies the contractive condition. In [4], Tomonari Suzuki has established a fixed point theorem in a compact metric space where the mapping $T$ satisfying a condition similarly to condition (II) of theorem 2 (see Lemma 5). Observe that any regular cone is always normal, Theorem 2 is established under a different and weaker condition when comparing with Lemma 4 and generalize the results of Lemma 5 from compact metric spaces to sequentially compact cone metric spaces.

Theorem 3. Let $(X, M, *)$ be a complete fuzzy metric space, where $*$ is defined by $a * b=a b$ for any $a, b \in[0,1]$ and $M$ a fuzzy set on $X^{2} \times(0, \infty)$ satisfying $\phi$-property. For a surjective function $f: X \rightarrow X$, if for any $x, y \in X, x \neq y$, the following inequality holds

$$
M(f(x), f(y), t) \geq M(x, y, \phi(t)),
$$

then $f$ has a fixed point on $X$. If inequality (II) is strict, then $f$ has a unique fixed point on $X$.

Proof. By choosing $y=f(x)$, we deduce from (II) that for any $x \in X$,

$$
M\left(f(x), f^{2}(x), t\right) \geq M(x, f(x), \phi(t)) \quad \text { (III) } \cdots
$$

Proceed by introduction on $n$, we have for any $n \in N$

$$
M\left(f^{n+1}(x), f^{n}(x), t\right) \geq M\left(f^{n}(x), f^{n-1}(x), \phi(t)\right) .
$$

For any $n>m$, we have

$$
\begin{aligned}
& M\left(f^{n}(x), f^{m}(x), t\right) \\
\geq & M\left(f^{n-1}(x), f^{m-1}(x), \phi(t)\right) \\
\geq & M\left(f^{m-1}(x), f^{m-2}(x), \phi^{2}(t)\right) \geq \cdots \\
\geq & M\left(f^{n-m-1}(x), f(x), \phi^{m-1}(t)\right) \\
\geq & M\left(f^{n-m}(x), x, \phi^{m}(t)\right)
\end{aligned}
$$

Observe that $M$ satisfies $\phi$-property, then

$$
\begin{aligned}
& \lim _{m \rightarrow \infty} M\left(f^{n}(x), f^{m}(x), t\right) \\
\geq & \lim _{m \rightarrow \infty} M\left(f^{n-m}(x), x, \phi^{m}(t)\right)=1,
\end{aligned}
$$

which shows that $\left\{f^{n}(x)\right\}$ is a fuzzy-Cauchy sequence. Since $(X, M, *)$ is complete, there exists $x_{0} \in X$, such that

$$
\lim _{n \rightarrow \infty} M\left(f^{n}(x), x_{0}, t\right)=1 .
$$
have

Then by (II) and the nondecreasing property of $M$, we

$$
\begin{aligned}
M\left(f^{n+1}(x), f\left(x_{0}\right), t\right) & \geq M\left(f^{n}(x), x_{0}, \phi(t)\right) \\
& \geq M\left(f^{n}(x), x_{0}, t\right),
\end{aligned}
$$

for any $x, y \in X, x \neq y$. Since

$$
\begin{gathered}
\lim _{n \rightarrow \infty} M\left(f^{n}(x), x_{0}, t\right)=1, \\
\lim _{n \rightarrow \infty} M\left(f^{n+1}(x), f\left(x_{0}\right), t\right)=1 .
\end{gathered}
$$

We therefore deduce

$$
\lim _{n \rightarrow \infty} f^{n}(x)=f\left(x_{0}\right)=x_{0},
$$

which shows $f$ has a fixed point on $X$.

If there exist $x_{0}, x_{0}^{\prime} \in X, x_{0} \neq x_{0}^{\prime}$ such that $f\left(x_{0}\right)=x_{0}, f\left(x_{0}^{\prime}\right)=x_{0}^{\prime}$, then by condition (II),

$$
\begin{aligned}
M\left(x_{0}, x_{0}^{\prime}, t\right) & =M\left(f\left(x_{0}\right), f\left(x_{0}^{\prime}\right), t\right) \\
& >M\left(x_{0}, x_{0}^{\prime}, \phi(t)\right) \geq M\left(x_{0}, x_{0}^{\prime}, t\right) .
\end{aligned}
$$

It is a contradiction, hence $x_{0}=x_{0}^{\prime}$. We have now proved the uniqueness which complete the proof.

Corollary 1. Let $(X, M, *)$ be a complete fuzzy metric space and $f: X \rightarrow X$ a bijective mapping, where $*$ is defined by $a * b=a b$ for any $a, b \in[0,1]$ and $M$ a fuzzy set on $X^{2} \times(0, \infty)$ satisfying $\phi$ property. If for any $x, y \in X, x \neq y$,

$$
M(f(x), f(y), \phi(t)) \leq M(x, y, t),
$$

then $f$ has a fixed point on $X$. If the above inequality is strict, then $f$ has a unique fixed point on $X$.

Proof. Since $f$ is bijective, $f^{-1}: X \rightarrow X$ exists and satisfies for any $x, y \in X, x \neq y$,

$$
\begin{aligned}
& M\left(f^{-1}(x), f^{-1}(y), t\right)=M(x, y, t) \\
& \geq M(f(x), f(y), \phi(t)) .
\end{aligned}
$$

By Theorem 3, we know $f^{-1}$ has fixed point, and the fixed point of $f^{-1}$ is the same as that of $f$, then $f$ has fixed point on $X$. If the inequality is strict, then the proof is the same as that in Theorem 3.

Corollary 2. Let $\left(X, M,{ }^{*}\right)$ be a complete fuzzy metric space, where $*$ is defined by $a * b=a b$ for any $a, b \in[0,1]$ and $M$ a fuzzy set on $X^{2} \times(0, \infty)$ satisfying $\tau$-property, and $f: X \rightarrow X$ a surjective mapping satisfying

$$
M(f(x), f(y), k t) \geq M(x, y, t),
$$

for any $x, y \in X, x \neq y, k \in(0,1)$. Then $f$ has a fixed point on $X$. If the inequality is strict, then $f$ has a unique fixed point on $X$.

Proof. Let $\phi(t)=1 k t, 0<k<1$, then by Theorem 3 we can easily propose the results of Corollary 2 . We omit the details.

Example 2. Assume $X=R, t \in(0, \infty)$, and define $M$ by

$$
M(x, y, t)=e^{\frac{-d(x, y)}{t}},
$$


clearly $M$ satisfies $\tau$-property. For any $f$ satisfies the conditions of Corollary 2, i.e.

$$
e^{\frac{-\mathrm{d}(f(x), f(y))}{k t}} \geq e^{\frac{-\mathrm{d}(x, y)}{t}},
$$

we have $\mathrm{d}(f(x), f(y)) \leq k \mathrm{~d}(x, y)$ for any $k \in(0,1)$, hence $f$ is a contraction mapping which has a fixed point on $X$.

In the following, we show an example to demonstrate the conditions in Corollary 2 are only sufficient condition, not necessary conditions.

Example 3. Assume $X=R, t \in(0, \infty)$, and define M by

$$
M(x, y, t)=e^{\mathrm{d}(x, y) t} .
$$

Obviously $M(x, y, t)$ is a fuzzy set which doesn't have $\tau$-property, hence it can't be judged by Corollary 3. But if $f$ is a contraction mapping, a fixed point still exist on $X$.

Theorem 4. Let $(X, M, *)$ be a complete fuzzy metric space, where $*$ is defined by $a * b=a b$ for any $a, b \in[0,1]$ and $M$ a fuzzy set on $X^{2} \times(0, \infty)$ satisfying $\phi$ - property. $F: X \rightarrow 2^{X}$ is a compact setvalued mapping, satisfies for any $x, y \in X$,

$$
H_{M}(F(x), F(y), t) \geq M(x, y, \phi(t)),
$$

then $F$ has a fixed point on $X$.

Proof. By the choice axioms (see [6]), there exists a single-valued function $f: X \rightarrow X$, such that $f(x) \in F(x)$ for any $x \in X$. Then for each $x_{1}, y_{1} \in X$, there exist

$x_{2}=f\left(x_{1}\right) \in F\left(x_{1}\right), y_{2}=f\left(y_{1}\right) \in F\left(y_{1}\right)$. By the definition of $H_{M}$, we have

$$
\begin{aligned}
& M\left(f\left(x_{1}\right), f\left(y_{1}\right), t\right)=M\left(x_{2}, y_{2}, t\right) \\
& \geq H_{M}\left(F\left(x_{1},\right) F\left(y_{1}\right), t\right) \geq M\left(x_{1}, y_{1}, \phi(t)\right) .
\end{aligned}
$$

Theorem 3 shows that $f$ has a fixed point $y_{0} \in X$, i.e. $y_{0}=f\left(y_{0}\right) \in F\left(y_{0}\right)$, which is also a fixed point of $F$ on $X$.

Corollary 3. Let $(X, M, *)$ be a complete fuzzy metric space and $M$ a fuzzy set on $X^{2} \times(0, \infty)$ satisfying $\tau$ - property. $F: X \rightarrow 2^{X}$ is a compact setvalued mapping satisfying for every $x, y \in X$,

$$
H_{M}(F(x), F(y), k t) \geq M(x, y, t), 0<k<1 .
$$

Then $F$ has a fixed point on $X$.

\section{Applications to Differential Equations}

This section is concerned with the proof of the existence and uniqueness of the solutions to the two-point ordinary differential equations by using the fixed point theorems obtained in Section 3. The following are the main results.

Theorem 5. Assume that $f: I \times R \rightarrow R$ is a continu- ous function. If there exists $\lambda>0$ such that the following inequalities

$$
0 \leq f(t, y)+\lambda y-[f(t, x)+\lambda x] \leq \lambda \omega(y-x),
$$

hold for any $x, y \in R$ with $y \geq x$, where $\omega$ is an $\omega$-function, then Problem(1) has a unique solution.

Proof. Problem (1) is equivalent to the integral equation

$$
u(t)=\int_{0}^{T} G(t, s)[f(s, u(s))+\lambda u(s)] \mathrm{d} s,
$$

where

$$
G(t, s)= \begin{cases}\frac{e^{\lambda(T+s-t)}}{e^{\lambda T}-1}, & 0 \leq s<t \leq T ; \\ \frac{e^{\lambda(s-t)}}{e^{\lambda T}-1}, & -0 \leq t<s \leq T .\end{cases}
$$

Define

$$
F: C([0, T], R) \rightarrow C([0, T], R)
$$

by

$$
(F u)(t)=\int_{0}^{T} G(t, s)[f(s, u(s))+\lambda u(s)] \mathrm{d} s .
$$

Note that if $u \in C([0, T], R)$ is a fixed point of $F$, then $u \in C([0, T], R)$ is a solution to Problem (1). Define a order relation in $C([0, T], R)$ by $u \leq v$ if and only if $u(t) \leq v(t)$ for every $t \in[0, T]$, for every $u, v \in C([0, T], R)$. Denote by $d(u, v)=\sup _{t \in[0, T]}|u(t)-v(t)|$ for any $u, v \in C([0, T], R)$ the distance in $C([0, T], R)$. For each $u>v$, by the left side of (IV),

$f(t, u)+\lambda u \geq f(t, v)+\lambda v$. Since $G(t, s)>0$, for each $(t, s) \in[0, T] \times[0, T], t \geq s$,

$$
\begin{aligned}
& (F u)(t)=\int_{0}^{T} G(t, s)[f(s, u(s))+\lambda u(s)] \mathrm{d} s \\
& \geq \int_{0}^{T} G(t, s)[f(s, v(s))+\lambda v(s)] \mathrm{d} s=(F v)(t),
\end{aligned}
$$

which shows that $F$ is monotone increasing. For any $u \geq v$, if $\varepsilon<d(u, v)<\varepsilon+\delta$, then

$$
\begin{aligned}
& d(F u, F v) \leq \sup _{t \in[0, T]}|(F u)(t)-(F v)(t)| \\
& \leq \sup _{t \in[0, T]} \int_{0}^{T} G(t, s) \\
& \cdot|f(s, u(s))+\lambda u(s)-f(s, v(s))-\lambda v(s)| \mathrm{d} s \\
& \leq \sup _{t \in[0, T]} \int_{0}^{T} G(t, s) \lambda \omega(u(s)-v(s)) \mathrm{d} s \\
& \leq \sup _{t \in[0, T]} \int_{0}^{T} G(t, s) \lambda \omega(d(u(s), v(s))) \mathrm{d} s .
\end{aligned}
$$

Since $\omega(t)$ is a increasing function, then 


$$
\begin{aligned}
& \omega(\mathrm{d}(u(s), v(s))) \leq \omega(\mathrm{d}(u, v)) \text { for } u>v, \text { and } \\
& \mathrm{d}(F u, F v) \leq \sup _{t \in[0, T]} \int_{0}^{T} G(t, s) \lambda \omega(\mathrm{d}(u(s), v(s))) \mathrm{d} s \\
& \leq \lambda \omega(\mathrm{d}(u, v)) \sup _{t \in[0, T]} \int_{0}^{T} G(t, s) \mathrm{d} s \\
& =\lambda \omega(\mathrm{d}(u, v)) \cdot \sup _{t \in[0, T]} \frac{1}{e^{\lambda T}-1}\left(\frac{1}{\lambda} e^{\lambda(T+s-t)}\left|+\frac{1}{\lambda} e^{\lambda(s-t)}\right|\right) \\
& \quad \leq \lambda \omega(\mathrm{d}(u, v)) \frac{1}{\lambda\left(e^{\lambda T}-1\right)}\left(e^{\lambda T}-1\right) \\
& \quad \leq \omega(\mathrm{d}(u, v)) .
\end{aligned}
$$

By the definition of $\omega(t)$, for each $\varepsilon>0$, there exists $M>0$ such that $\varepsilon<\mathrm{d}(u, v)<M, \quad \omega(\mathrm{d}(u, v))<\varepsilon$, let $\delta=M-\varepsilon>0$, hence $\varepsilon<\mathrm{d}(u, v)<\varepsilon+\delta, \quad \omega(\mathrm{d}(u, v))<\varepsilon$. It demonstrates $\mathrm{d}(F u, F v)<\varepsilon$. By Lemma 3, $F$ has a unique fixed point, and $\lim _{n \rightarrow \infty} F^{n}\left(u_{0}\right)=u$ for each

$u_{0} \in C([0, T], R), u$ is the fixed point of $F$, i.e. the solution of Problem (1).

Assume $\alpha(t)$ is a lower solution of Problem (1), we can prove as Theorem 3.1 in [13] to obtain the uniqueness of the solution.

Remark 8. Contrasted with some related results in [13-15], the conditions in Theorem 5 is relatively clearer.

Theorem 6. Assume that $f: I \times R \rightarrow R$ is a continuous function. If there exists $\lambda>0$ such that for any $x, y \in R$ with $y \geq x$, the following inequalities

$$
\begin{aligned}
0 & \leq f(t, y)-f(t, x)+g(t, y)-g(t, x) \\
& \leq \lambda \eta(y-x)
\end{aligned}
$$

hold, where $\eta$ is an $\omega$-function, then the solution of Problem (2) exists.

Proof. Problem (2) is equivalent to the following integral equation

$$
x(t)=x_{0}+\int_{0}^{t} f(s, x(s)) \mathrm{d} s+\int_{0}^{t} g(s, x(s)) \mathrm{d} s .
$$

Define

$$
F: C([0, T] ; R) \rightarrow C([0, T] ; R)
$$

by

$$
(F u)(t)=x_{0}+\int_{0}^{t} f(s, u(s))+g(s, u(s)) \mathrm{d} s,
$$

for any $u(t, x) \in C([0, T] ; R)$. Note that $u \in C([0, T] ; R)$ is a fixed point of $F$, then $u \in C([0, T] ; R)$ is a solution of Problem (2). For $u, v \in C([0, T] ; R)$, we define $u \leq v$ if and only if $u(t) \leq v(t)$ for any $t \in[0, T]$. Denote $d(u, v)=\sup _{t \in[0, T]}|u(t)-v(t)|$, for $u, v \in C([0, T] ; R)$.

Then by (V), for any $u>v$,

$$
\begin{gathered}
f(t, u) \geq f(t, v), \\
f(t, u(s))+g(t, u(s)) \geq f(t, v(s))+g(t, v(s)),
\end{gathered}
$$

which implies

$$
\begin{aligned}
& (F u)(t)=x_{0}+\int_{0}^{t} f(s, u(s))+g(s, u(s)) \mathrm{d} s \\
& \geq x_{0}+\int_{0}^{t} f(s, v(s))+g(s, v(s)) \mathrm{d} s=(F v)(t),
\end{aligned}
$$

and

$$
\begin{aligned}
& \mathrm{d}(F u, F v) \leq \sup _{t \in[0, T]}|(F u)(t)-(F v)(t)| \\
& \leq \sup _{t \in[0, T]} \mid \int_{0}^{t}[f(s, u(s))-f(s, v(s))] \mathrm{d} s \\
& +\int_{0}^{t}[g(s, u(s))-g(s, v(s))] \mathrm{d} s \mid \\
& \leq \sup _{t \in[0, T]}\left|\int_{0}^{T} \lambda \eta(u(s)-v(s)) \mathrm{d} s\right| \quad(0<k<1) \\
& \leq \sup _{t \in[0, T]}\left|\int_{0}^{T} \lambda \eta(\mathrm{d}(u, v)) \mathrm{d} s\right| .
\end{aligned}
$$

By the definition of function $\omega$, let $\varepsilon_{1}=\varepsilon T \lambda>0$, there exists $M>0$, such that

$\varepsilon_{1}<\mathrm{d}(u, v)<\varepsilon T \lambda, \omega(\mathrm{d}(u, v))<\varepsilon_{1}$, there exists $\delta>0$, such that $M=\varepsilon+\delta$, then $\varepsilon_{1}<\mathrm{d}(u, v)<\varepsilon_{1}+\delta$, $\eta(\mathrm{d}(u, v))<\varepsilon_{1}$ and

$$
\sup _{t \in[0, T]}\left|\int_{0}^{T} \lambda \eta(\mathrm{d}(u, v)) \mathrm{d} s\right| \leq \sup _{t \in[0, T]} \int_{0}^{T} \lambda \varepsilon d s \leq 2 T \lambda \varepsilon_{1}=\varepsilon .
$$

By Lemma 3, $F$ has a unique fixed point, and $\lim _{n \rightarrow \infty} F^{n}\left(u_{0}\right)=u$ for any $u_{0} \in C([0, T] ; R), u$ is a fixed point of $F$, which is also a solution of Problem (2). The proof is complete.

Define $\theta: R \rightarrow R$ satisfying for any $u, v \in C([0, T] ; R)$,

$$
\begin{aligned}
& \theta(\lambda u(s))-\theta(\lambda v(s)) \\
& \geq f(s, u(s))-\lambda u(s)-(f(s, v(s))-\lambda v(s)),
\end{aligned}
$$

then we have the following theorem:

Theorem 7. Let $(X, M, *)$ be a complete fuzzy metric space, $M(x, y, t)=e^{\frac{-d(x, y)}{t}}$. If the following conditions hold:

1) For any $x, y \in R, x \neq y, k \in(0,1)$,

$$
M(\theta(x), \theta(y), k t) \geq M(x, y, t) ;
$$

2) For any $x \leq y$,

$$
0 \leq f(t, y)+\lambda y-[f(t, x)+\lambda x],
$$

then the solution of Problem (1) is unique.

Proof. By example 2, while $M(x, y, t)=e^{\frac{-\mathrm{d}(x, y)}{t}}$, a 
mapping satisfying the above conditions is a contraction mapping, i.e. $\theta$ is a contraction mapping. Then we can proceed the proof with the same arguments as that in Theorem 5.

Remark 9. If we replace condition (1) by the inequality in Example 2 or Example 3 as well as the corresponding expression of $M$, then Theorem 7 can also make sure the uniqueness of the solution of Problem (1).

Define $h: R \rightarrow R$ satisfying for any

$$
\begin{aligned}
u, v \in C([0, T] & ; R), \\
& h(\lambda u(s))-h(\lambda v(s)) \\
\geq & {[f(s, u(s))-(f(s, v(s))]} \\
+ & {[g(s, u(s))-[g(s, v(s))],}
\end{aligned}
$$

then we have the following theorem:

Theorem 8. Let $\left(X, M,{ }^{*}\right)$ be a complete fuzzy metric space, $M(x, y, t)=e^{\frac{-\mathrm{d}(x, y)}{t}}$. If the following two conditions hold:

1) for any $x, y \in R, x \neq y$ and $k \in(0,1)$,

$$
M(h(x), h(y), k t) \geq M(x, y, t) ;
$$

2) for any $x \leq y$,

$$
0 \leq f(t, y)-f(t, x), 0 \leq g(t, y)-g(t, x),
$$

then the solution of Problem (2) exists.

Proof. By Example 2, while $M(x, y, t)=e^{\frac{-\mathrm{d}(x, y)}{t}}$, a mapping satisfying the conditions above is a contraction mapping, hence $h$ is a contraction mapping. Then we can proceed the proof with the same arguments as that in Theorem 6 and complete the proof.

\section{Conclusion}

The paper is devoted to several new types of fixed point theorems in different spaces such as cone metric spaces and fuzzy metric spaces together with their applications. We have also proved the existence and uniqueness of the solutions to two classes of two-point ordinary differential equation problems by using these obtained fixed point theorems.

\section{Acknowledgements}

The authors of this paper would like to appreciate the referee's helpful comments and valuable suggestions which have essentially improved this paper. This work is supported by the National Natural Science Foundation (11071109) of People’s Republic of China.

\section{REFERENCES}

[1] K. Deimling, “Nonlinear Functional Analysis,” Springer-
Verlag, Berlin, 1985. doi:10.1007/978-3-662-00547-7

[2] C. J. Zhang, "Set-Valued Analysis and Its Applications to Economics,” The Science Press, Beijing, 2004.

[3] W. Walter, "Remarks on a Paper by F. Browder about Contraction,” Nonlinear Analysis, Vol. 5, 1981, pp. 21-25. doi:10.1016/0362-546X(81)90066-3

[4] T. Suzuki, "A New Type of Fixed Point Theorem in Metric Spaces,” Nonlinear Analysis, Vol. 71, 2009, pp. 53135317. doi:10.1016/j.na.2009.04.017

[5] A. Meir and E. Keeler, "A Theorem on Contraction Mappings," Journal of Mathematical Analysis and Applications, Vol. 28, 1969, pp. 326-329. doi:10.1016/0022-247X(69)90031-6

[6] T. Cardinali and P. Rubbioni, "An Extension to Multifunctions of the Keeler-Meir's Fixed Point Theorem," Fixed Point Theory, Vol. 7, No. 1, 2006, pp. 23-36.

[7] L.-G. Huang and X. Zhang, "Cone Metric Spaces and Fixed Point Theorems of Contractive Mappings,” Journal of Mathematical Analysis and Applications, Vol. 332, No. 2, 2007, pp. 1468-1476.

[8] A. George and P. Veeramani, "On Some Result in Fuzzy Metric Space,” Fuzzy Sets and Systems, Vol. 64, 1994, pp. 395-399. doi:10.1016/0165-0114(94)90162-7

[9] S. Sedghi, I. Altunb and N. Shobe, "Coupled Fixed Point Theorems for Contractions in Fuzzy Metric Spaces," Nonlinear Analysis, Vol. 72, 2010, pp. 1298-1304. doi:10.1016/j.na.2009.08.018

[10] X.-H. Zhu and J.-Z. Xiao, "Note on "Coupled Fixed Point Theorems for Contractions in Fuzzy Metric Spaces'," Nonlinear Analysis, Vol. 74, 2011, pp. 5475-5479. doi:10.1016/j.na.2011.05.034

[11] S. S. Zhang, "Fixed Point Theorems of Mappings on Probabilistic Metric Spaces with Applications," Scientia Sinca (Series A), Vol. 11, 1983.

[12] J. Rodriguez-Lopez and S. Romaguera, "The Hausdorff Fuzzy Metric on Compact Sets," Fuzzy Sets and Systems, Vol. 147, No. 2, 2004, pp. 273-283.

[13] A. Amini-Harandi and H. Emami, “A Fixed Point Theorem for Contraction Type Maps in Partially Ordered Metric Spaces and Application to Ordinary Differential Equations,” Nonlinear Analysis, Vol. 72, 2010, pp. 2238-2242. doi:10.1016/j.na.2009.10.023

[14] T. G. Bhaskar and V. Lakshmikantham, "Fixed Point Theorems in Partially Ordered Metric Spaces and Applications,” Nonlinear Analysis, Vol. 65, 2006, pp. 13791393. doi:10.1016/j.na.2005.10.017

[15] J. Harjani and K. Sadarangani, “Generalized Contractions in Partially Ordered Metric Spaces and Applications to Ordinary Differential Equations," Nonlinear Analysis, Vol. 72, 2010, pp. 1188-1197. doi:10.1016/j.na.2009.08.003

[16] J. Harjani and K. Sadarangani, "Fixed Point Theorems for Weakly Contractive Mappings in Partially Ordered Sets," Nonlinear Analysis, Vol. 71, 2009, pp. 3403-3410. doi:10.1016/j.na.2009.01.240 